Mi Jin Lee', Tae-Yong Shin ${ }^{2}$, Chang Hee Lee ${ }^{3}$, Jun dong Moon ${ }^{4}$, Sang Gyun Roh ${ }^{5}$, Chan Woong Kim ${ }^{6}$, Hyo Eun Park ${ }^{7}$, Seon Hee Woo ${ }^{8}$, Seung Joon Lee ${ }^{9}$, Seung Lyul Shin ${ }^{10}$, Young Taeck Oh ${ }^{11}$, Yong Su Lim ${ }^{12}$, Jae Young Choe ${ }^{1}$, Sang-Hoon $\mathrm{Na}^{13}$, Sung Oh Hwang ${ }^{14}$; on behalf of the Steering Committee of 2020 Korean Guidelines for Cardiopulmonary Resuscitation and Emergency Cardiovascular Care

\footnotetext{
'Department of Emergency Medicine, Kyungpook National University School of Medicine, Daegu, Korea ${ }^{2}$ Department of Emergency Medicine, Asan Chungmu General Hospital, Asan, Korea

${ }^{3}$ Department of Emergency Medical Technician, Namseoul University, Cheonan, Korea

${ }^{4}$ Department of Emergency Medical Service, College of Health \& Nursing, Kongju National University,

Gongju, Korea

${ }^{5}$ Department of Emergency Medical Services, Sun Moon University, Asan, Korea

${ }^{6}$ Department of Emergency Medicine, Chung-Ang University College of Medicine, Seoul, Korea

${ }^{7}$ Division of Cardiology, Department of Internal Medicine, Healthcare System Gangnam Center, Seoul

National University Hospital, Seoul, Korea

${ }^{8}$ Department of Emergency Medicine, The Catholic University of Korea College of Medicine, Seoul, Korea

${ }^{9}$ National Medical Emergency Center, National Medical Center, Seoul, Korea

${ }^{10}$ Department of Emergency Medicine, Inha University College of Medicine, Incheon, Korea

${ }^{11}$ Department of Emergency Medicine, Seoul National University College of Medicine, Seoul, Korea

${ }^{12}$ Department of Emergency Medicine, Gachon University College of Medicine, Incheon, Korea

${ }^{13}$ Department of Internal Medicine, Seoul National University College of Medicine, Seoul, Korea

${ }^{14}$ Department of Emergency Medicine, Yonsei University Wonju College of Medicine, Wonju, Korea
}

\section{MAJOR CHANGES}

This chapter periodically updates the evidence-based education and system implementation (EIT) guidelines with regard to reflecting evolving best training strategies on how best to implement successful resuscitation programs in Korea. A major change in the EIT section of the 2020 Korean guidelines for cardiopulmonary resuscitation (CPR) reflects an extension of the revised scope from the previous exclusive focus on education to the inclusion of the current system implementation. As the educational approaches are the core links among their implementation into practice, we provide the components of education in resuscitation at a more advanced level, based upon the survival environment. This extension highlights the importance of social competence for CPR education and a sudden cardiac death management system as a key element in constructing and realizing the survival environment, as newly described in the 2020 Korean CPR guidelines. The EIT Task Force conducted 16 systemic reviews applying the GRADE (Grading of Recommendation, Assessment, Development, and Evaluation) guidance., ${ }^{1,2}$ The EIT of the present
elSSN: 2383-4625

Received: 7 March 2021

Revised: 7 March 2021

Accepted: 28 March 2021

Correspondence to: Mi Jin Lee Department of Emergency Medicine, Kyungpook National University School of Medicine, 130 Dongdeok-ro, Junggu, Daegu 41944, Korea

E-mail:emmam@knu.ac.kr ORCID

https://orcid.org/0000-0002-3773-8047

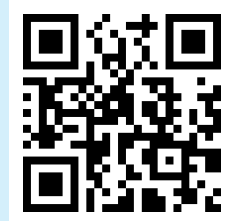

How to cite this article:

Lee MJ, Shin TY, Lee CH, Moon JD, Roh SG, Kim CW, Park HE, Woo SH, Lee SJ, Shin SL, Oh YT, Lim YS, Choe JY, Na SH, Hwang SO; on behalf of the Steering Committee of 2020 Korean Guidelines for Cardiopulmonary Resuscitation and Emergency Cardiovascular Care. 2020 Korean Guidelines for Cardiopulmonary Resuscitation. Part 9. Education and system implementation for enhanced chain of survival. Clin Exp Emerg Med 2021;8(S):S116-S124. https://doi. org/10.15441/ceem.21.029

This is an Open Access article distributed under the terms of the Creative Commons Attribution Non-Commercial License (https:// creativecommons.org/licenses/by-nc/4.0/). 
guidelines describes the consensus on scientific evidence and treatment recommendations of the 16 major issues that have been reviewed by the International Liaison Committee on Resuscitation in 2020 and revised to be compatible with the current status in South Korea.

The updated topics include the comprehensive factors to construct integrated survival outcomes based on the latest technology-enhanced training to teach resuscitation (e.g., self-directed CPR training, blended learning, contactless edu-technology, gamified learning, and virtual reality environment, etc.), the use of social networks and mobile phone technologies to notify volunteer bystanders, and strengthening the role for emergency dispatchers. In addition, early warning scoring and the rapid response team (RRT) for preventing cardiac arrest in hospitals, high-performed paramedic CPR with ongoing reinforced and continuous quality improvement regarding advanced resuscitation, and conditional recommendation of the termination of resuscitation (TOR) rules that should be reflected in the prehospital and emergency medical system (EMS) are updated..$^{3-5}$

\section{CORE PYRAMID OF THE NEW STRATEGIES}

Survival after cardiac arrest is determined by distinct factors depending on the circumstances in which sudden cardiac death occurs. Specific to out-of-hospital cardiac arrest (OHCA), rapid defibrillation and bystander CPR by the witness is a critical determinant of survival. For in-hospital cardiac arrest (IHCA), the same factors, as well as the early recognition of the possibility of cardiac arrest, are crucial. Patients usually show signs and symptoms of deterioration before cardiac arrest event. The rapid response system is a program designed to improve the safety of patients whose condition is worsening. ${ }^{6}$ Thus, defining the most effective means of resuscitation education is similar to setting and achieving the goal of enhancing the survival rate in cardiac arrest patients.

The chain of survival has been extended to the Utstein formula for survival, which includes the prevention of cardiac arrest. ${ }^{5,7}$ The addition of spaced learning (education or retraining frequently separated by longer periods of time) or booster training to resuscitation courses based on new education methodologies and technological advancement seems to be more effective skill retention than massed learning (education provided close in the whole time). ${ }^{8,9}$ In the event of an emerging infectious disease such as the pandemic coronavirus disease 2019 (COVID-19), several contactless education platforms have been developed and commercialized in Korea, taking into account the local accessibility and circumstances that hinder face-to-face training. The CO-
VID-19 guidelines are updated based on evolving knowledge and experience in the epidemic. ${ }^{10,11}$

It is very important to teach the technical skills of performing resuscitation at all levels. The specific educational needs of these different target groups (from citizens, first responder to health care clinicians) exist in series, depending on individual and organizational CPR competency requirements. The required competency level determines the CPR training interval, frequency, need for retraining, and programs level (from basic to advanced life support [ALS] levels). Understanding the clinical personnel's exposure or general experience on OHCA outcomes may inform training strategies. It also focuses on ALS training for paramedics with relatively little experience in managing cardiac arrest for high-quality resuscitation treatment.

The system guidelines for cardiac arrest treatment and their implementation suggest a novel TOR model that can be applied in the prehospital field and early phase, in addition to managing the RRT for preventing cardiac arrest in hospital. This guideline also proposes an integrated management of cardiac arrests in the community with monitoring strategies for the respective cardiac arrest registry. The following are the contents of the core pyramid of ESI for the enhanced chain of survival (Fig. 1).

\section{SUMMARY OF THE AGREEMENTS ON SCIENTIFIC EVIDENCE AND TREATMENT RECOMMENDATIONS}

These EIT guidelines focus on all steps in the continuum of care to improve cardiac arrest survival; to increase the proportion of patients with OHCA who receive immediate bystander CPR and early defibrillation; to prevent IHCA by RRT implementation, and to measure the resuscitation team performance and resuscitation outcomes. To enhance the prognosis of survival after cardiac arrest, it is necessary to have a workforce in the local community, resuscitation instructors and instructor trainers, guideline developers, and an integrated system of relevant organizations.

The chain of survival emphasizes early recognition of cardiac arrest/activation of the emergency response system, high-quality CPR, early defibrillation, advanced resuscitation support, and post-cardiac arrest care/rehabilitation. For OHCA, the main contributors are effective bystander CPR and early use of public access defibrillator (PAD). Emergency response system development, layperson and dispatcher training in the recognition of cardiac arrest, public education promotion, widespread PAD availability, and tele-communicator instructions are all important components of this step in OHCA setting. In addition, the following should be conducted for IHCAs: prevention and early response to cardiac 


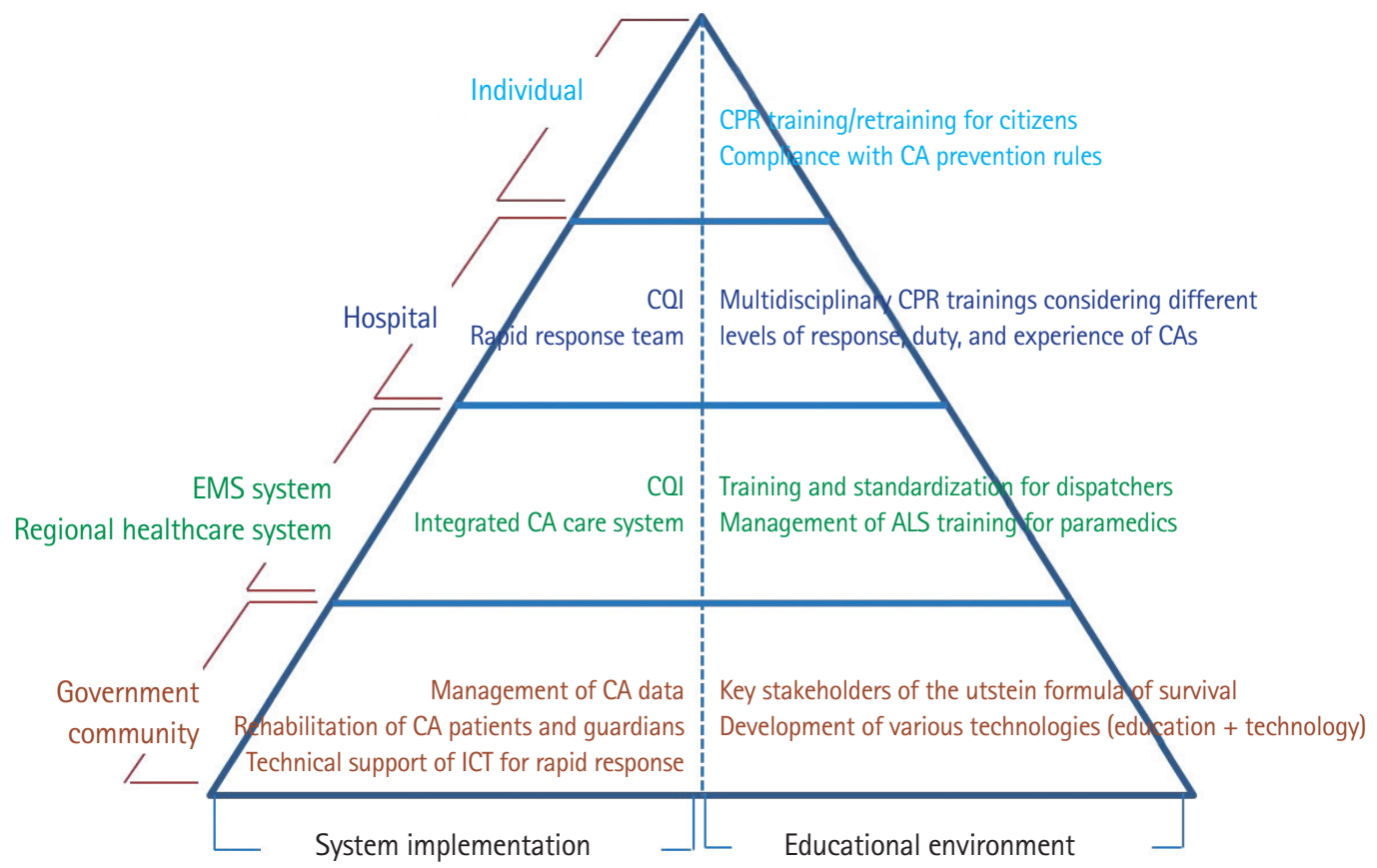

Fig. 1. Core pyramid of education and system implementation for enhanced chain of survival. CPR, cardiopulmonary resuscitation; $C A$, cardiac arrest; CQI, continuous quality improvement; EMS, emergency medical services; ALS, advanced life support; ICT, information and communication technology.

arrest via the preemptive management of the rapid response system, efficient management of the CPR team, control of ALS training for hospital staff, and quality improvement activities for an enhanced chain of survival related to CPR in hospitals. ${ }^{7}$ Lastly, while long-term recovery after cardiac arrest indicates good neurological prognosis, it includes cognitive, physical and psychological rehabilitation and recovery that targets the patients, family, and healthcare providers. ${ }^{12,13}$

\section{Integrated management to promote CPR implementation in community}

As part of the prevention and treatment of cardiac arrest in the entire country or local community, the system level takes into account the following factors: the proportion of witnessed bystander CPR and PAD use, ${ }^{14,15}$ intervention of prehospital or in-hospital therapeutic hypothermia (or targeted temperature management), and use of automated mechanical CPR devices or feedback devices. $^{16,17}$ The 2020 resuscitation guidelines recommend that key indicators be evaluated, while specific goals to enhance the performance status have been set for the EMS system, local community, and medical institutions providing cardiac arrest treatment (Class Ila, Level B-NR). However, as the current situation in South Korea involves separate management of OHCAs and IHCAs, it may be reasonable for integrated social managements to implement strategies for enhanced survival outcomes and increasing bystander CPR.

\section{Data monitoring and establishment of cardiac arrest registries}

A cardiac arrest registry is the essence of measurement. Local communities and EMS systems should establish and monitor the performance data on the social contributors of cardiac arrest survival and related processes. This involves data registration research on all steps of cardiac arrest and patient outcomes at regional community or nationwide levels. ${ }^{11,18}$ Continuous measurements will determine if implementing changes leads to improvements. To achieve this, a database should be constructed to integrate registries at the local government, firefighting department, hospitals, national emergency medical center, and the Ministry of Health and Welfare data. In South Korea, the Korea Disease Control and Prevention Agency conducts research to collect and analyze the data on OHCA cases that are then applied in the national statistics. In the US, patient registration systems based on emergency centers have been established. One is the registry for IHCA (Get With The Guidelines-Resuscitation) ${ }^{19}$ and the others are statistics for OHCA (Cardiac Arrest Registry to Enhance Survival or Resuscitation Outcomes Consortium Cardiac Registry). ${ }^{20}$ Data from these systems are essential in local community liaisons, monitoring quality improvement activities, managing quality indicators for quality improvement, and successfully enhancing the cardiac arrest survival rate in the local community. 


\section{Reinforce the roles of dispatchers with ongoing training and continuous quality improvement}

The role of dispatchers or tele-communicators that connect the active EMS system and the general public in emergencies is the critical link in the chain of survival of OHCA. CPR performed by witnesses with the guidance of dispatchers (telephone-assisted CPR or dispatcher-assisted CPR) increases the rate of witness resuscitations in adult OHCA patients. However, it is difficult to accurately determine whether cardiac arrest has occurred in the field over the phone. Dispatchers accurately identify cardiac arrests over the phone in about 70\% of cases. ${ }^{21}$ If the suspected diagnosis of cardiac arrest is incorrect, the patients will receive inappropriate chest compression or CPR will not begin. Programs that improve the quality of dispatcher-assisted CPR and the feedback from physicians to dispatchers have improved the outcome of cardiac arrest patients. To be able to deliver the CPR guidance over the phone, dispatchers should complete a specific educational program. ${ }^{22}$ Specific training of dispatchers on how to deliver telephone-assisted CPR can lead to improved cardiac arrest recognition, reduction of misinterpretation of agonal respiration, increased rate of patients receiving chest compressions, and a shorter time to compression.

We recommend that dispatchers use a standard algorithm to determine cardiac arrest situations through emergency calls (Class I, Level C-LD). To maximize the rate of resuscitation via telephone-assisted CPR, efforts should be made towards the development and active use of guidelines for dispatchers and scripted questions (to determine if a victim is unresponsive with abnormal breathing), and quality improvement through evaluating the debriefing after such telephone instructions and how to receive accurate feedback. ${ }^{23,24}$ It is suggested that any dispatchers receive education for potential cardiac arrest situations that include the following: 1) recognizing a cardiac arrest; 2) encouraging the willingness of rescuers to perform CPR and notifying their safety checks; 3 ) providing instructions to perform chest compression-only CPR and to use an automated external defibrillator $(A E D) ; 4)$ helping the EMS to reach the location of the incident. $^{25,26}$

\section{Basic life support including AED training}

Education about communication during basic life support (BLS) is important in order to overcome barriers that rescuers might experience in performing CPR and use of the AED. There are three main barriers: personal factors (emotional barriers, most often panic, but also socio-economic factors); CPR knowledge (skill deficits, fear of damage or doing something wrong); and procedural issues (e.g., recognition of cardiac arrest, location of AED). ${ }^{27-29}$
Addressing these barriers and enablers to starting CPR might increase the willingness to help those in a life-threatening situation.

Treatment recommendation has not changed since 2010 (strong recommendation, low-certainty evidence). To increase willingness to perform CPR and use AED, laypersons should receive training. CPR training should include the recognition of gasping or abnormal respiration as a sign of cardiac arrest. The EMS dispatcher should provide CPR instruction to callers who report cardiac arrest. If unwilling or unable to perform mouth-to-mouth ventilation, rescuers should be instructed to continue compression-only CPR.

\section{CPR feedback devices in training}

Use of feedback devices during resuscitation training can be effective in improving CPR performance. Since the last search in 2015, based on the evidence of simulation studies and certain clinical studies regarding the use of feedback devices in CPR education, these treatment recommendations have not changed. We suggest the use of feedback devices that provide directive feedback on chest compression rate, depth, decompression, and hand position for improving the CPR performance. If a feedback device is not available, it may be helpful to use a tonal guidance including music or metronome for feedback to only improve the chest compression rate (Class Ila, Level B-R).

\section{Experience of EMS practitioners and exposure to OHCA}

The survival of prehospital cardiac arrests is influenced by a range of factors, and one of which is to provide high-quality resuscitation. Appropriate provision of prehospital resuscitation is an important element in determining good neurological prognosis from $\mathrm{OHCA}^{30}$ Understanding the impact of ongoing exposure or experience on patient outcomes from OHCA can help with staffing and training strategies. The exposure of EMS personnel is associated with improved short-term survival outcomes. ${ }^{31-33}$ In order to increase the level of CPR experience, the national agency or local community should build organically interconnected systems to educate EMS providers, paramedics, relevant educational institutions, and interhospital emergency medical directors through participation in CPR situation or supervising their procedure-focused advanced training.

Unlike the medical staff in hospitals that frequently encounter cardiac arrest patients, field EMS practitioners, including paramedics that provide treatment to OHCAs, are relatively less exposed to actual cardiac arrest management cases. It is reasonable for EMS systems to monitor provider exposure to resuscitation to implement strategies to address issues of low exposure (Class Ilb, 
Level C-LD). Thus, for the effective treatment of OHCA patients, EMS directors are advised to do as follows: 1) manage the experience or exposure of EMS personnel in resuscitation situations; 2) develop strategies, if possible, to address the problem of lack of resuscitation experience, or form a resuscitation team to include members with experience, especially with a recent resuscitation.

\section{Multidisciplinary CPR trainings in hospital at different levels of response and duty}

High-quality resuscitation training is essential for health care providers (HCPS) at all levels from BLS to ALS, for children and/or adults, depending on their workplace requirement for CPR competencies. Considering the medical staff at hospitals that work in departments with little exposure to cardiac arrest cases, a plan for certified resuscitation education should be developed. A meta-analysis of related studies has shown that resuscitation teams with one or more members having previous participation in an ALS course improve survival outcomes. It is reasonable for HCP to take an adult ALS course or equivalent training. At least one member of the in-hospital resuscitation team should be included in the certified ALS course, while medical staff and HCPs working in a department with higher CPR incidence should provide ALS training in addition to BLS as part of the certified or complementary education (Class Illb, Level C-LD).

\section{Revised simulation to teach resuscitation}

Simulation in resuscitation training is widely used and is a wellestablished educational strategy. It facilitates contextualized learning relating to learner's reality. Learning CPR can be supported by the use of smartphones, tablets, etc. by using apps and social media, as well as feedback devices. These learning modalities may be teacher-independent. They improve retention and facilitate competency assessment in CPR. The 2020 guideline recommends using e-learning as part of the blended learning approach to reduce the time for conventional face-to-face education in advanced resuscitation. This requires comprehensive management of instructor-led lectures, simulation practices, and testing of conventional education (Class Ila, Level C-LD).

Taking into account these characteristics, learning objectives need to be defined for participating individuals or CPR teams, as well as choosing the adequate equipment. The use of high-fidelity manikins for continuous ALS programs can be effective for learners at training centers with available infrastructure and trained personnel (Class Ila, Level C-LD). If high-fidelity manikins are not available, the use of low-fidelity manikins that are suitable for the standard programs to achieve the educational goals may be considered (Class IIb, Level C-LD). Additionally, the latest tablet-based simulation apps combined with the use of low-fidelity manikins may be suitable alternative at reasonable cost for simulation during resuscitation training.

\section{Use of technology-enhanced education modules}

As cardiac arrest cases are, by nature, not frequently experienced, it is difficult to develop alternative cases for educational purposes. However, advances in technology have allowed us to use new resources for teaching CPR over the past few years. ${ }^{34,35}$ Access to medical content has never been easier because of the ubiquitous availability of the internet. Gamified learning (e.g., virtual and augmented reality, smartphones or tablet apps simulating monitors) can be turned into a compression feedback device or become a simulated monitor. Serious games on mobile devices engage CPR learners differently than traditional classroom lessons. ${ }^{35-38}$ Virtual learning environments are recommended to be used for pre-course e-learning, as part of a blended learning approach, or self-learning independent of time and location for all levels of courses. ${ }^{39,40}$ Therefore, these devices and programs should be included in future CPR educational approaches and combined with face-to-face education (Class IIb, Level B-NR). It might be possible to integrate training related to simulated patients in virtual or augmented reality as a complementary tool to

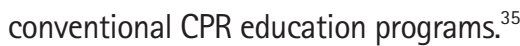

In unique circumstances that hinder face-to-face training, such as a pandemic or national disaster, conventional education and evaluation of knowledge and performance using general CPR training programs can be replaced with such tools as virtual reality, augmented reality, or web-based contact platforms (Class IIb, Level C-LD). Several contactless platforms for resuscitation education (e.g., self-directed educator, high-frequency methods that apply for the resuscitation quality improvement program) are commercially available in South Korea and other countries. It could be used as a method of complementary education or retraining for $\mathrm{HCP}$ as well as noncontact resuscitation education for the general public. ${ }^{41}$

\section{Effective skill retention and retraining methodology} Spaced learning (education or retraining separated by longer periods of time) seems to be more effective in CPR training than traditional massed instruction (education provided close together in time) (Fig. 2) ${ }^{8,17,42-44}$ BLS skills decline within 3 to 12 months after initial CPR training, but evidence suggests that more frequent retraining improves CPR skills, responders' confidence, and willingness to perform CPR (Fig. 3) (Class Illb, Level C-LD). ${ }^{45}$ 


\section{Early warning score and RRTs for IHCA}

Recently, rapid response systems (e.g., RRT or medical emergency team) have been operating in South Korea since 2020. RRT has been installed as a type of constant in-hospital monitoring system to prevent the occurrence of cardiac arrest through early intervention before risk of deterioration and cardiac arrest. ${ }^{6,46} \mathrm{An}$ early warning scoring system and an active in-hospital protocol using the alerting system should be developed alongside the activities of RRT to prevent the incidence of IHCA in adult and pediatric populations. For hospitalized adults, RRT can be effective in reducing the incidence of cardiac arrest, especially in general wards (Class Ila, Level C-LD).

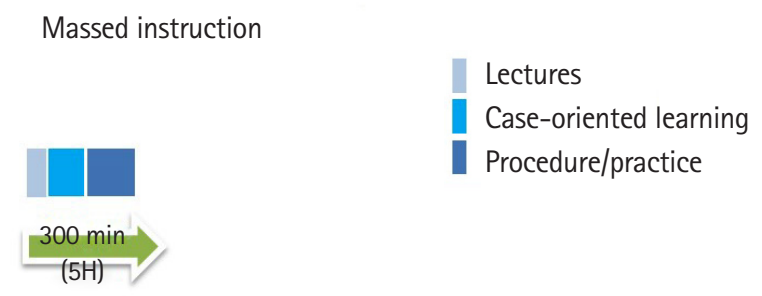

Spaced learning

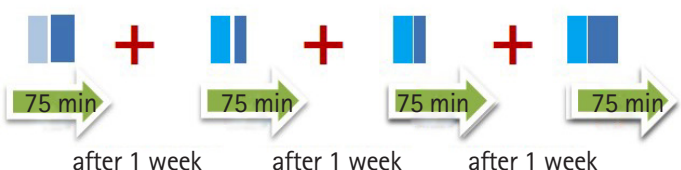

Fig. 2. Example of module differences between massed instruction and spaced learning.

\section{Rapid alerting events by mobile phone technologies for OHCA}

To alert trained lay rescuers of events requiring CPR or PAD use, the use of mobile phone technology by emergency dispatcher systems is reasonable. Notification of lay rescuers via a smartphone app or text message is associated with shorter bystander response times. As these technologies become more ubiquitous, they are likely to play an expanding role in the chain of survival. However, it is recommended that the responders in close proximity to patients with suspected cardiac arrest are advised to ask for help by sending their global positioning system location via smartphone applications and social network systems (text messages) with prior consent (Class Ila, Level B-R).

\section{Multimodal approach of TOR rules in prehospital and EMS system}

We suggest a list of TOR rules for adult cardiac arrest patients (prehospital and emergency department settings). ${ }^{47}$ This aims to

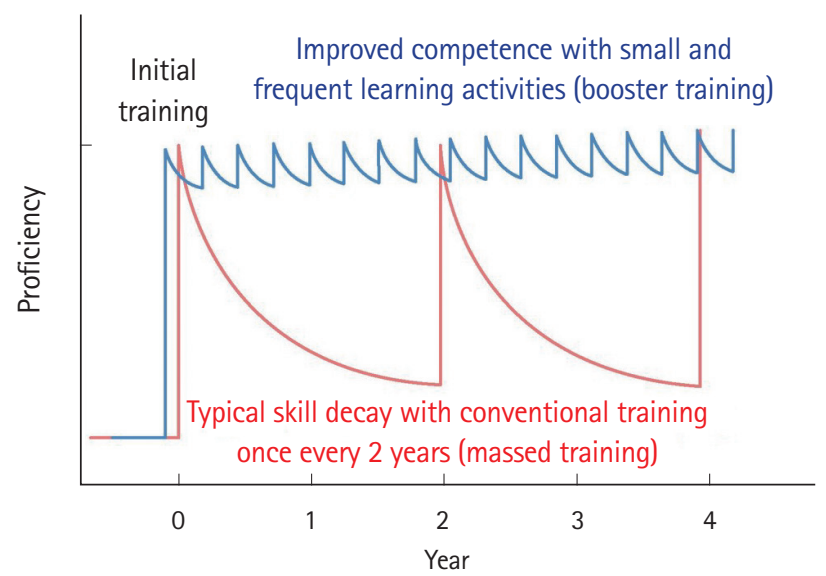

Fig. 3. Scheme of booster training for small and frequent learning.

Table 1. Comparison of TOR rules

\begin{tabular}{|c|c|c|c|c|c|}
\hline TOR rules & Witness status & Initial prehospital rhythm & Prehospital shock & Prehospital ROSC & Others \\
\hline \multicolumn{6}{|l|}{ Based on prehospital criteria } \\
\hline International BLS rule $\mathrm{e}^{51}$ & Not witnessed by EMT & - & No prehospital shock & No prehospital ROSC & - \\
\hline Goto's TOR rule ${ }^{49}$ & Not witnessed by bystander & Initial non-shockable rhythm & - & No prehospital ROSC & - \\
\hline KoCARC TOR rule $I^{52}$ & Not witnessed by EMT & Asystole in prehospital conditions & No prehospital shock & No prehospital ROSC & - \\
\hline KoCARC TOR rule II & Not witnessed by EMT & - & No prehospital shock & No prehospital ROSC & Age $>60 \mathrm{yr}$ \\
\hline New TOR model $1^{50}$ & Not witnessed by bystander & Asystole in the field & - & No prehospital ROSC & - \\
\hline \multicolumn{6}{|l|}{ Based on prehospital and ED } \\
\hline SOS-KANTO's TOR rule $e^{48}$ & Not witnessed by bystander & Asystole in the field & - & - & Asystole in the hospital \\
\hline New TOR model $2^{50}$ & Not witnessed by bystander & - & - & No prehospital ROSC & Asystole in the hospital \\
\hline
\end{tabular}


enable more efficient distribution and use of EMS resources available in the community, to address the issue of increased risk of HCPs and poor CPR quality during transport, and to prevent unnecessary hospital transport. Futile resuscitation could also cause a risk of infection transmission in OHCA patients with emerging infectious diseases. ${ }^{10}$ The conventional TOR rules have been validated based on a patient registry in the U.S. and in Asia (Japan and South Korea). The KoCARC (Korean Cardiac Arrest Research Consortium) reports and various regional multicenter studies have been conducted. ${ }^{48-52}$ Based on different stages, ten TOR rules have, so far, been developed that can be applied in the prehospital fields ${ }^{49,53}$ or in emergency department setting $\mathrm{s}^{54}$ (Table 1). ${ }^{48-53}$ Most TOR rules comprise a stage-by-stage assessment system with consideration of not a single criterion but of multiple conditions, including unwitnessed status, asystole rhythm in the field, no prehospital shock, or ROSC is not achieved prior to arrival at the hospital.

We conditionally recommend the multimodal approach of TOR rules to assist clinicians in deciding whether to discontinue resuscitation efforts out of hospital or to transport to the emergency department with ongoing CPR (Conditional recommendation, Level C-LD). Thus, these prehospital TOR rules are considered a complementary tool in determining whether to terminate resuscitation in the field or to continue resuscitation until arrival at the hospital..$^{50}$ Notably, the sole use of the TOR rules should be avoided in deciding to terminate or postpone resuscitation. As the current Emergency Medical Service Acts and laws do not allow the decision to be made solely by paramedics in South Korea, the final decision should be confirmed by the emergency medical director.

\section{CONFLICT OF INTEREST}

No potential conflict of interest relevant to this article was reported.

\section{ACKNOWLEDGMENTS}

This study was supported by a grant (2020E330300) of the Korean Disease Control and Prevention Agency funded by the Ministry of Health and Welfare, Republic of Korea.

We thank Ms. So Yeong Kim (EMT) for her assistance with administrative affairs and Mr. Myung Ha Kim for his assistance with literature searches for updating Korean Guidelines for cardiopulmonary resuscitation. We also thank the Korean Association of Cardiopulmonary Resuscitation (KACPR) for supporting the process of proofreading.

\section{REFERENCES}

1. Greif $R$, Bhanji $F$, Bigham BL, et al. Education, implementation, and teams: 2020 international consensus on cardiopulmonary resuscitation and emergency cardiovascular care science with treatment recommendations. Circulation 2020; 142(16_suppl_1):S222-83.

2. Morrison $\sqcup$, Gent $L M$, Lang $E$, et al. Part 2: evidence evaluation and management of conflicts of interest. 2015 American Heart Association guidelines update for cardiopulmonary resuscitation and emergency cardiovascular care. Circulation 2015;132(18 Suppl 2):S368-82.

3. Berg KM, Cheng A, Panchal AR, et al. Part 7: systems of care. 2020 American Heart Association Guidelines for cardiopulmonary resuscitation and emergency cardiovascular care. Circulation. 2020;142(16_suppl_2):S580-604.

4. Cheng $A$, Magid DJ, Auerbach $M$, et al. Part 6: resuscitation education science. 2020 American Heart Association Guidelines for cardiopulmonary resuscitation and emergency cardiovascular care. Circulation 2020;142(16_suppl_2):S551-79.

5. Cheng A, Nadkarni VM, Mancini MB, et al. Resuscitation education science: educational strategies to improve outcomes from cardiac arrest. A scientific statement from the American Heart Association. Circulation 2018;138:e82-122.

6. Avis E, Grant L, Reilly E, Foy M. Rapid response teams decreasing intubation and code blue rates outside the intensive care unit. Crit Care Nurse 2016;36:86-90.

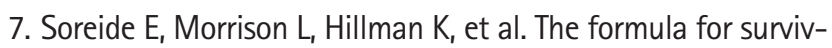
al in resuscitation. Resuscitation 2013;84:1487-93.

8. Anderson R, Sebaldt A, Lin Y, Cheng A. Optimal training frequency for acquisition and retention of high-quality $C P R$ skills: a randomized trial. Resuscitation 2019;135:153-61.

9. Nishiyama C, Iwami T, Murakami Y, et al. Effectiveness of simplified 15-min refresher BLS training program: a randomized controlled trial. Resuscitation 2015;90:56-60.

10. Cho JW, Jung H, Lee MJ, et al. Preparedness of personal protective equipment and implementation of new CPR strategies for patients with out-of-hospital cardiac arrest in the COVID-19 era. Resusc Plus 2020;3:100015.

11. Kim C, Yeo IH, Kim JK, et al. Confirmation of COVID-19 in out-of-hospital cardiac arrest patients and postmortem management in the emergency department during the COVID-19 outbreak. Infect Chemother 2020;52:562-72.

12. Ro YS, Shin SD, Song KJ, Hong SO, Kim YT, Cho SI. Bystander cardiopulmonary resuscitation training experience and self-efficacy of age and gender group: a nationwide community survey. Am J Emerg Med 2016;34:1331-7. 
13. Yang HJ, Kim GW, Cho GC, Tak YJ, Chung SP, Hwang SO. Part 8. Cardiopulmonary resuscitation education: 2015 Korean Guidelines for Cardiopulmonary Resuscitation. Clin Exp Emerg Med 2016;3(Suppl):S66-8.

14. Hansen SM, Hansen CM, Folke F, et al. Bystander defibrillation for out-of-hospital cardiac arrest in public vs residential locations. JAMA Cardiol 2017;2:507-14.

15. Pollack RA, Brown SP, Rea T, et al. Impact of bystander automated external defibrillator use on survival and functional outcomes in shockable observed public cardiac arrests. Circulation 2018;137:2104-13.

16. Katipoglu B, Madziala MA, Evrin $T$, et al. How should we teach cardiopulmonary resuscitation? Randomized multi-center study. Cardiol J 2019 Sep 30. https://doi.org/10.5603/CJ.a2019.0092.

17. Lin $Y$, Cheng A, Grant VJ, Currie GR, Hecker KG. Improving CPR quality with distributed practice and real-time feedback in pediatric healthcare providers: a randomized controlled trial. Resuscitation 2018;130:6-12.

18. Kim YT, Shin SD, Hong SO, et al. Effect of national implementation of utstein recommendation from the global resuscitation alliance on ten steps to improve outcomes from out-ofhospital cardiac arrest: a ten-year observational study in Korea. BMJ Open 2017;7:e016925.

19. Bradley SM, Liu W, Chan PS, et al. Duration of resuscitation efforts for in-hospital cardiac arrest by predicted outcomes: insights from Get With The Guidelines--Resuscitation. Resuscitation 2017;113:128-34.

20. Virani SS, Alonso A, Benjamin EJ, et al. Heart disease and stroke statistics: 2020 update. A report from the American Heart Association. Circulation 2020;141:e139-596.

21. Dami F, Heymann E, Pasquier M, Fuchs V, Carron PN, Hugli 0. Time to identify cardiac arrest and provide dispatch-assisted cardio-pulmonary resuscitation in a criteria-based dispatch system. Resuscitation 2015;97:27-33.

22. Tanaka Y, Taniguchi J, Wato Y, Yoshida Y, Inaba H. The continuous quality improvement project for telephone-assisted instruction of cardiopulmonary resuscitation increased the incidence of bystander CPR and improved the outcomes of outof-hospital cardiac arrests. Resuscitation 2012;83:1235-41.

23. Kurz MC, Bobrow BJ, Buckingham J, et al. Telecommunicator cardiopulmonary resuscitation: a policy statement from the American Heart Association. Circulation 2020;141:e686-700.

24. Lewis M, Stubbs BA, Eisenberg MS. Dispatcher-assisted cardiopulmonary resuscitation: time to identify cardiac arrest and deliver chest compression instructions. Circulation 2013; 128:1522-30.

25. Clegg GR, Lyon RM, James S, Branigan HP, Bard EG, Egan GJ.
Dispatch-assisted CPR: where are the hold-ups during calls to emergency dispatchers? A preliminary analysis of caller-dispatcher interactions during out-of-hospital cardiac arrest using a novel call transcription technique. Resuscitation 2014; 85:49-52.

26. Vaillancourt $C$, Charette $M$, Kasaboski $A$, et al. Cardiac arrest diagnostic accuracy of 9-1-1 dispatchers: a prospective multi-center study. Resuscitation 2015;90:116-20.

27. Choe MSP, Lee MJ. National survey of awareness and training experience of automated external defibrillator. J Korean Soc Emerg Med 2019;30:301-8.

28. Lee MJ, Hwang SO, Cha KC, Cho GC, Yang HJ, Rho TH. Influence of nationwide policy on citizens' awareness and willingness to perform bystander cardiopulmonary resuscitation. Resuscitation 2013;84:889-94.

29. Vaillancourt $C$, Kasaboski $A$, Charette $M$, et al. Barriers and facilitators to CPR training and performing CPR in an older population most likely to witness cardiac arrest: a national survey. Resuscitation 2013;84:1747-52.

30. Perkins GD, Jacobs IG, Nadkarni VM, et al. Cardiac arrest and cardiopulmonary resuscitation outcome reports: update of the Utstein Resuscitation Registry Templates for out-of-hospital cardiac arrest. A statement for healthcare professionals from a task force of the International Liaison Committee on Resuscitation (American Heart Association, European Resuscitation Council, Australian and New Zealand Council on Resuscitation, Heart and Stroke Foundation of Canada, InterAmerican Heart Foundation, Resuscitation Council of Southern Africa, Resuscitation Council of Asia); and the American Heart Association Emergency Cardiovascular Care Committee and the Council on Cardiopulmonary, Critical Care, Perioperative and Resuscitation. Circulation 2015;132:1286-300.

31. Dyson K, Bray JE, Smith K, Bernard S, Straney L, Finn J. Paramedic exposure to out-of-hospital cardiac arrest resuscitation is associated with patient survival. Circ Cardiovasc Qual Outcomes 2016;9:154-60.

32. Tuttle JE, Hubble MW. Paramedic out-of-hospital cardiac arrest case volume is a predictor of return of spontaneous circulation. West J Emerg Med 2018;19:654-9.

33. Weiss $N$, Ross $E_{1}$ Cooley $C$, et al. Does experience matter? Paramedic cardiac resuscitation experience effect on out-ofhospital cardiac arrest outcomes. Prehosp Emerg Care 2018; 22:332-7.

34. Bonnetain $E$, Boucheix JM, Hamet M, Freysz M. Benefits of computer screen-based simulation in learning cardiac arrest procedures. Med Educ 2010;44:716-22.

35. Leary M, McGovern SK, Chaudhary Z, Patel J, Abella BS, Blew- 
er AL. Comparing bystander response to a sudden cardiac arrest using a virtual reality CPR training mobile app versus a standard CPR training mobile app. Resuscitation 2019;139: 167-73.

36. Ringh $M$, Fredman $D$, Nordberg P, Stark T, Hollenberg J. Mobile phone technology identifies and recruits trained citizens to perform CPR on out-of-hospital cardiac arrest victims prior to ambulance arrival. Resuscitation 2011;82:1514-8.

37. Berglund $E_{1}$ Claesson $A$, Nordberg $P$, et al. A smartphone application for dispatch of lay responders to out-of-hospital cardiac arrests. Resuscitation 2018;126:160-5.

38. Stroop R, Kerner T, Strickmann B, Hensel M. Mobile phonebased alerting of CPR-trained volunteers simultaneously with the ambulance can reduce the resuscitation-free interval and improve outcome after out-of-hospital cardiac arrest: a German, population-based cohort study. Resuscitation 2020; 147:57-64.

39. Lehmann R, Lutz T, Helling-Bakki A, Kummer S, Huwendiek S, Bosse HM. Animation and interactivity facilitate acquisition of pediatric life support skills: a randomized controlled trial using virtual patients versus video instruction. BMC Med Educ 2019;19:7.

40. Lehmann $R$, Thiessen $C$, Frick $B$, et al. Improving pediatric basic life support performance through blended learning with webbased virtual patients: randomized controlled trial. J Med Internet Res 2015;17:e162.

41. Dudzik LR, Heard DG, Griffin RE, et al. Implementation of a low-dose, high-frequency cardiac resuscitation quality improvement program in a community hospital. Jt Comm J Qual Patient Saf 2019;45:789-97.

42. Ernst KD, Cline WL, Dannaway DC, et al. Weekly and consecutive day neonatal intubation training: comparable on a pediatrics clerkship. Acad Med 2014;89:505-10.

43. Patocka $C$, Cheng $A$, Sibbald $M$, et al. A randomized education trial of spaced versus massed instruction to improve acquisition and retention of paediatric resuscitation skills in emergency medical service (EMS) providers. Resuscitation 2019; 141:73-80.

44. Sullivan NJ, Duval-Arnould J, Twilley M, et al. Simulation exercise to improve retention of cardiopulmonary resuscitation priorities for in-hospital cardiac arrests: a randomized controlled trial. Resuscitation 2015;86:6-13.

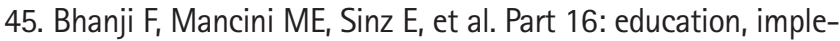
mentation, and teams. 2010 American Heart Association Guidelines for cardiopulmonary resuscitation and emergency cardiovascular care. Circulation 2010;122(18 Suppl 3):S92033.

46. Al-Qahtani S, Al-Dorzi HM, Tamim HM, et al. Impact of an intensivist-led multidisciplinary extended rapid response team on hospital-wide cardiopulmonary arrests and mortality. Crit Care Med 2013;41:506-17.

47. Morrison $\sqcup$, Eby $D$, Veigas $P V$, et al. Implementation trial of the basic life support termination of resuscitation rule: reducing the transport of futile out-of-hospital cardiac arrests. Resuscitation 2014;85:486-91.

48. SOS-KANTO 2012 Study Group. A new rule for terminating resuscitation of out-of-hospital cardiac arrest patients in Japan: a prospective study. J Emerg Med 2017;53:345-52.

49. Goto Y, Funada A, Maeda T, Okada H, Goto Y. Field termination-of-resuscitation rule for refractory out-of-hospital cardiac arrests in Japan. J Cardiol 2019;73:240-6.

50. Lee DE, Lee MJ, Ahn JY, et al. New termination-of-resuscitation models and prognostication in out-of-hospital cardiac arrest using electrocardiogram rhythms documented in the field and the emergency department. J Korean Med Sci 2019;34: e134.

51. Rotering VM, Trepels-Kottek S, Heimann K, Brokmann JC, Orlikowsky T, Schoberer M. Adult "termination-of-resuscitation" (TOR)-criteria may not be suitable for children: a retrospective analysis. Scand J Trauma Resusc Emerg Med 2016;24:144.

52. Yoon JC, Kim YJ, Ahn S, et al. Factors for modifying the termination of resuscitation rule in out-of-hospital cardiac arrest. Am Heart J 2019:213:73-80.

53. Glober NK, Tainter CR, Abramson TM, Staats K, Gilbert G, Kim D. A simple decision rule predicts futile resuscitation of outof-hospital cardiac arrest. Resuscitation 2019;142:8-13.

54. Morrison $\sqcup$, Verbeek PR, Vermeulen MJ, et al. Derivation and evaluation of a termination of resuscitation clinical prediction rule for advanced life support providers. Resuscitation 2007; 74:266-75. 